\title{
Fractional Describing Function Analysis of PWPF Modulator
}

\author{
Xinsheng Wang, ${ }^{1,2}$ Danwei Wang, ${ }^{2}$ Senqiang Zhu, ${ }^{2}$ and Eng Kee $\mathrm{Poh}^{3}$ \\ ${ }^{1}$ Department of Control Science and Engineering, Harbin Institute of Technology at Weihai, Weihai 264209, China \\ ${ }^{2}$ EXQUISITUS, Centre for E-City, School of Electrical and Electronic Engineering, \\ Nanyang Technological University, Singapore 639798 \\ ${ }^{3}$ DSO National Laboratories, Singapore 117510
}

Correspondence should be addressed to Xinsheng Wang; wangxswh@126.com

Received 9 November 2012; Accepted 8 January 2013

Academic Editor: Clara Ionescu

Copyright (C) 2013 Xinsheng Wang et al. This is an open access article distributed under the Creative Commons Attribution License, which permits unrestricted use, distribution, and reproduction in any medium, provided the original work is properly cited.

Pulse-width pulse-frequency (PWPF) modulators are widely used in spacecraft thruster control. Their dynamic characteristic is still lack of effective analysis tools. This paper presents a fractional describing function method to describe the frequency characteristics of PWPF. A frequency-dependent gain and phase shift are clearly described by fractional-order expression, and the fractional-order behaviors depict the nonlinear properties of PWPF modulators. This fractional describing function method can also be applied to other kinds of modulators.

\section{Introduction}

Spacecrafts commonly deploy thrusters as actuators for attitude control [1]. As thrusters work on on/off switching, there is a conversion from continuous desired torque control command to an on/off signal for spacecraft thrusters. There are two common approaches for thruster activation. The simplest converter is a bang-bang controller which is, however, vulnerable to noise. Several improved controllers have been proposed, such as bang-bang controller with dead zone or time-optimal bang-bang controller [2]. Another kind of the converter is pulse modulators, which produce a pulse command sequence to the thruster valves by adjusting pulse width and/or pulse frequency according to the level of input [3]. Among the known pulse modulators, the pulse-width pulse-frequency (PWPF) modulators are the most common and enjoy advantages over bang-bang control systems [4]. But the inherent nonlinearity of PWPF has inhibited the dynamic analysis of attitude control system.

Describing function method is a well-known analysis tool for a kind of nonlinear system with certain structure. If the output signal of a nonlinear device can be approximated by the fundamental harmonics, the fundamental harmonics can be used to define the frequency characteristics of the nonlinear element and is called a describing function [5].
The describing function method is primarily used to analyze the stability and limit cycle of nonlinear control systems and is inherently approximate. In the last decades, many new criteria were established to improve the accuracy. These new types of describing function methods include areamatching method, root-mean-square method, and corrected RMS method [3].

Recently, fractional calculus has been increasingly applied to mechanical systems, electricity, and bioengineering [6, 7]. Fractional calculus studies derivatives and integrals of fractional order. It is shown that fractional-order system works more accurately than integer-order system $[8,9]$. With the development of computational tools, more and more fractional-order models and controllers are studied [10, 11]. Noticeably, the describing function method is also considered in the framework of nonlinear fractional-order systems [12, 13]. In this paper, fractional describing function will be introduced to describe the dynamics of PWPF actuators.

\section{Pulse-Width Pulse-Frequency Modulator}

Pulse modulators are commonly used in thruster control of fuel valves. There are various kinds of pulse modulators, such as pulse-width modulator, pulse-frequency modulator, 


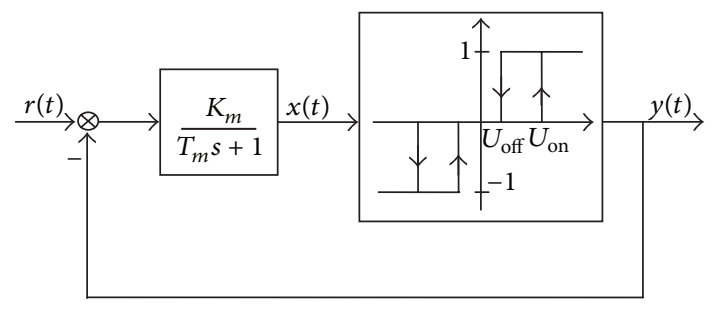

Figure 1: Structure of PWPF modulator.

TABLE 1: Static characteristic variables of PWPF modulator.

\begin{tabular}{lc}
\hline On time & $T_{\text {on }}=-T_{m} \ln \left(1-\frac{h}{U_{\text {on }}-K_{m}(C-U)}\right)$ \\
Off time & $T_{\text {off }}=-T_{m} \ln \left(1-\frac{h}{K_{m} C-U_{\text {off }}}\right)$ \\
Modulator frequency & $f=\frac{1}{T_{\text {on }}+T_{\text {off }}}$ \\
Duty cycle & $\mathrm{DC}=\frac{T_{\text {on }}}{T_{\text {on }}+T_{\text {off }}}$ \\
$\begin{array}{l}\text { Equivalent internal } \\
\text { deadband }\end{array}$ & $C_{d}=\frac{U_{\text {on }}}{K_{m}}$ \\
$\begin{array}{l}\text { Equivalent internal } \\
\text { saturation level }\end{array}$ & $C_{s}=1+\frac{U_{\text {off }}}{K_{m}}$ \\
\hline
\end{tabular}

pseudo-rate modulator, and pulse-width pulse-frequency (PWPF) modulator $[14,15]$. PWPF modulator is preferred for its operation has almost linear input/output relationship [16, 17]. A PWPF modulator mainly comprises two components: a first-order lag filter and a Schmitt trigger inside a feedback loop, as shown in Figure 1. A Schmitt trigger is an on-off relay with a dead zone and hysteresis. It differs from bangbang controller in that there are two thresholds: one on-value $U_{\text {on }}$ and one off-value $U_{\text {off }}$. These values define a hysteresis as $h=U_{\text {on }}-U_{\text {off }}$. The output of the Schmitt trigger is compared with the reference signal, and the error is fed to the first-order filter whose output is the input of the Schmitt trigger. PWPF modulator operates in a quasilinear mode by modulating the width of the output pulses and the distance between them simultaneously. And it can produce pulses in two directions: positive and negative pulses.

With a constant input $C$, the PWPF modulator drives the thruster valve with an on-off pulse sequence having a nearly linear duty cycle. The time interval in which the modulator has a nonzero output is denoted $T_{\text {on }}$, and the time interval with a zero output is denoted $T_{\text {off }}$. Static characteristics variables of PWPF are collected in Table 1. These variables are considered in the modulator design. Paper [18] shows the relationship between the static characteristics of PWPF modulator and selection of its parameters.

The static analysis of PWPF modulator shows that it operates near linear to the constant input over a large range between the deadband $C_{d}$ and saturation level $C_{s}$. The modulator's operations are independent from the spacecraft's parameters and allow easy parameter tuning, especially when there are different requirements through different phases of operation. In addition, this modulator has the superiority in fuel consumption and pointing accuracy in the presence of vibrations. However, attitude control systems usually operate on dynamic mode, and PWPF modulators will introduce phase lag to the attitude control systems, which can cause instability. Dynamic analysis is necessary for the attitude control system design. But as to this nonlinear device, effective tools are limited. This work will propose the use of fractional-order describing function method and develops some useful techniques.

\section{Derivation of Fractional Describing Function}

Describing function method is an approximation method for analyzing nonlinear dynamics, because only the first harmonic of the output of a nonlinear element is considered. But here describing function method is beneficial for PWPF modulator analysis for the following reasons. Firstly, the firstorder filter which is in series with the nonlinear element serves as a low-pass filter in PWPF modulator. Secondly, the attitude control system is a high-order system, where high harmonics are attenuated substantially. In this section, the PWPF modulator is considered as a single device and its fractional describing function is developed.

3.1. Describing Function of Hysteresis. A nonlinear element to a sinusoidal input $x(t)=X \sin (\omega t)$ in general does not generate a sinusoidal output, but the output $y(t)$ is periodic. If the nonlinearity is symmetrical with respect to the variation around zero, the output signal can be decomposed to the Fourier series:

$$
\begin{aligned}
y(t) & =\sum_{n=1}^{\infty}\left(A_{n} \cos n \omega t+B_{n} \sin n \omega t\right) \\
& =\sum_{n=1}^{\infty} Y_{n} \sin \left(n \omega t+\varphi_{n}\right),
\end{aligned}
$$

where

$$
\begin{gathered}
A_{n}=\frac{1}{\pi} \int_{0}^{2 \pi} y(t) \cos n \omega t d(\omega t), \\
B_{n}=\frac{1}{\pi} \int_{0}^{2 \pi} y(t) \sin n \omega t d(\omega t), \\
Y_{n}=\sqrt{A_{n}^{2}+B_{n}^{2}}, \\
\varphi_{n}=\tan ^{-1} \frac{A_{n}}{B_{n}},
\end{gathered}
$$

and the frequency of the fundamental harmonic is the same frequency as the input with amplitude $Y_{1}$ and phase shift $\varphi_{1}$ :

$$
\begin{aligned}
y_{1}(t) & =A_{1} \cos \omega t+B_{1} \sin \omega t \\
& =Y_{1} \sin \left(\omega t+\varphi_{1}\right) .
\end{aligned}
$$

With the assumption that the amplitude of fundamental harmonic is much larger than the amplitude of other harmonics, the describing function is defined as the complex ratio of 
the fundamental harmonic component of the output and the input, that is,

$$
N(X, \omega)=\frac{Y_{1}}{X} e^{j \varphi_{1}},
$$

where $N(X, \omega)$ is the describing function of the nonlinear element, $X$ is the sinusoidal input amplitude, $\omega$ is the frequency of input sinusoid, $Y_{1}$ is the amplitude of the fundamental harmonic, $Y_{1} / X$ is the describing function gain, and $\varphi_{1}$ is the describing function phase.

Imposing this harmonic balance principle, $N(X, \omega)$ can be used as frequency characteristic of nonlinear element to analyze the dynamics of a linear closed-loop system. The Schmitt trigger in PWPF modulator exhibits hysteresis nonlinearity, whose describing function of hysteresis can be deduced as shown above directly:

$$
N(X)=\frac{4}{\pi X} \sqrt{1-\left(\frac{h}{X}\right)^{2}}-j \frac{4 h}{\pi X^{2}} .
$$

Remark 1. The describing function defined above is based on fundamental harmonic equivalence. The influence of high order harmonics can be considered as system uncertainty.

\subsection{Frequency Characteristics of PWPF. The PWPF modula-} tor is a unit in the attitude control system. Considering, the frequency characteristics of PWPF as a whole is convenient for analysis and design of the attitude control system. In the structure of Figure 1, the frequency characteristic of the firstorder filter is

$$
L(j \omega)=\frac{K_{m}}{1+j \omega T_{m}} .
$$

Then the frequency characteristic of the PWPF closedloop system is

$$
\begin{aligned}
N N(X, \omega)= & \left(\frac{4 K_{m}}{\pi X} \sqrt{1-\left(\frac{h}{X}\right)^{2}}-j \frac{4 h K_{m}}{\pi X^{2}}\right) \\
& \times\left(\left(1+\frac{4 K_{m}}{\pi X} \sqrt{1-\left(\frac{h}{X}\right)^{2}}\right)+j\left(\omega T_{m}-\frac{4 h K_{m}}{\pi X^{2}}\right)\right)^{-1} .
\end{aligned}
$$

If the describing function is independent of frequency $\omega$, it is plotted with varying nonlinear input $X$. But when it is a function of both amplitude $X$ and frequency $\omega$, certain $\omega$ values are selected to view the plot of frequency characteristics. Consequently, this kind of plot lacks frequency dependent information. For control system dynamic analysis, information about how the amplitude and phase of a nonlinear element change with frequency is important because the nonlinear part usually adds phase lag to control system, which would destroy close-loop stability of the control system.

Now, a fractional describing function is introduced to give a direct relationship between the nonlinear characteristic

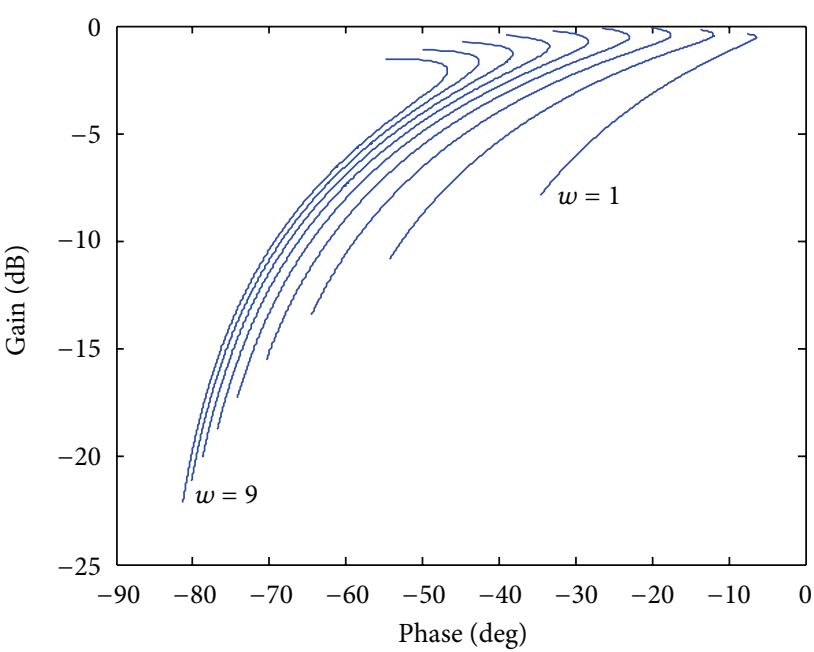

Figure 2: Nichols plot of $N N(X, \omega)$.

and frequency. For illustration purpose, set the parameters of PWPF as follows: $K_{m}=7.46, T_{m}=1.33, U_{\text {on }}=0.45$, and $U_{\text {off }}=0.25$. Figure 2 shows the Nichols plot of the closedloop describing function $N N(X, \omega)$. It can be seen that with the amplitude $X$ changing from 0.5 to 10 the magnitude of $N N(X, \omega)$ decreases, and the phase lag increases. To show the characteristic with frequency, several curves corresponding with different frequencies are plotted. Though it is clear that higher frequency induces larger phase lag, but the curves are separated and cannot show continuous information about frequency.

To reveal the relationship between the describing function and the frequency of interest, the real part and imaginary part of $N N(X, \omega)$ are studied, respectively. For attitude control, the interested frequency is relatively low. Figures 3 and 4 show the log-log plots of the real part and imaginary part of $N N(X, \omega)$ versus the exciting frequency $\omega$, respectively, where the nonlinear input $X$ is from 0.5 to 1 . Approximately, the curves can be considered as straight lines. Then a fractional-order behavior is investigated.

The fractional-order behavior is described by power functions, so it is called fractional describing function. The new function can be written as

$$
\operatorname{FNN}(X, \omega)=-a \omega^{-b}-j c \omega^{-d}, \quad\{a, b, c, d\} \in \mathrm{R}^{+} .
$$

Figure 3 shows that the real part of $N N(X, \omega)$ can be considered as a constant, that is, $b=0$ and $-0.9<a<$ -0.88 . Figure 4 shows that the imaginary part of $N N(X, \omega)$ is fractional with $-0.75<d<-0.7$ and $0.11<c<$ 0.13 . These values will vary with system parameters, but the fractional property remains the same. It can be seen that the range of parameters $\{a, b, c, d\}$ does not vary much. We can choose one set of values to simulate the frequency characteristic of the PWPF modulator. Here, we set the values $\{a, b, c, d\}=\{-0.9,0,0.12,-0.74\}$. With the help of available fractional calculator software $[19,20]$, frequency characteristic diagrams can be plotted. Figure 5 shows the Bode diagram of $\operatorname{FNN}(X, \omega)$. It predicts a phase lag of 


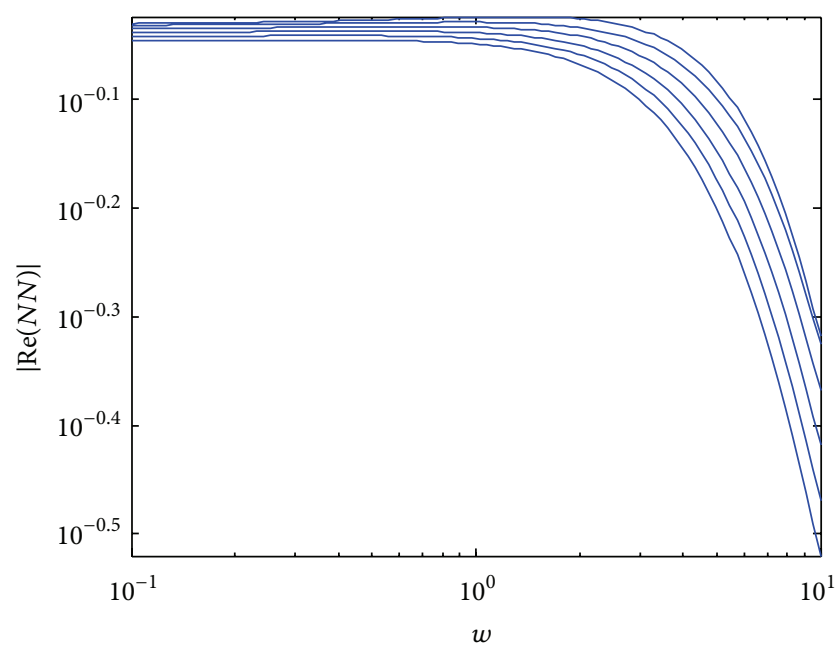

FIgURE 3: Log-log plots of $|\operatorname{Re}(N N)|$.

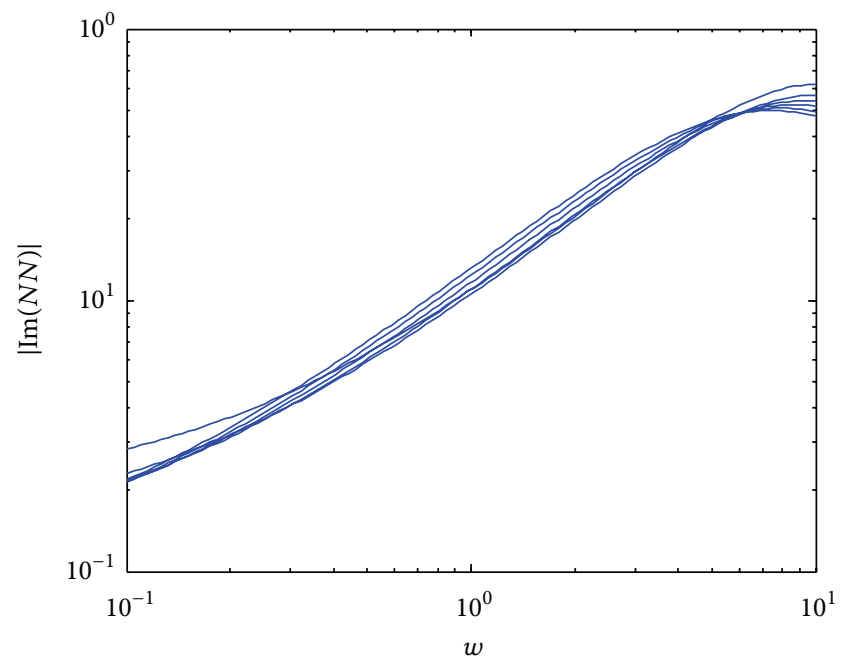

FIgURE 4: Log-log plots of | $\operatorname{Im}(N N) \mid$.

about 10 degrees to 25 degrees between frequency $1 \mathrm{rad} / \mathrm{s}$ to $5 \mathrm{rad} / \mathrm{s}$, which relates well with the analysis result in [3]. This information is crucial to the stability analysis of attitude control system.

Remark 2. The deduced fractional describing function is useful for system stability analysis for it is considered around the crossover frequency.

\section{Conclusions}

PWPF modulator is a nonlinear actuator in spacecraft attitude control system. The nonlinear dynamic behavior of PWPF modulator is investigated by the fractional describing function in this paper. The nonlinear element of PWPF is a Schmitt trigger, and its frequency characteristic can be described by describing function, and the fractional behaviors are caused by nonlinear element in PWPF. The frequency

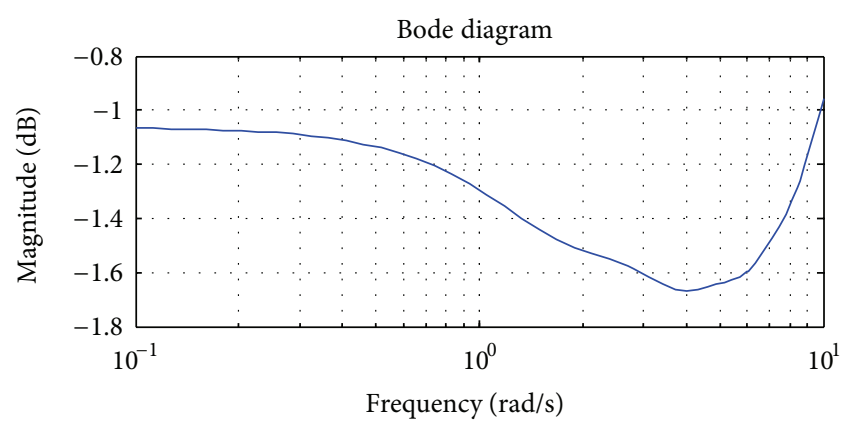

(a)

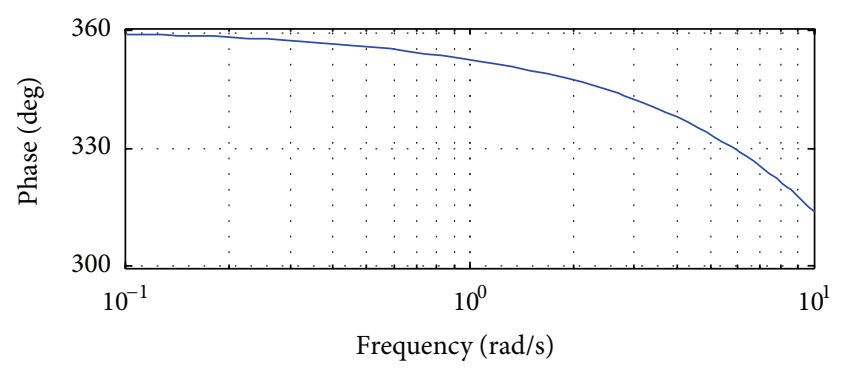

(b)

FIgURE 5: Bode diagram of $F N N(X, \omega)$.

characteristic of the actuator is frequency dependent. The loglog plots of the real part and imaginary part of a modulator clearly reveal the fractional-order behavior. The imaginary component is described by fractional-order power function over a certain frequency range. With fractional calculus, these frequency-dependent gain and phase information can be plotted in Bode diagram and used for control system design. Furthermore, the fractional describing function method should be an effective tool for other kinds of modulators.

\section{Acknowledgments}

This work is funded in part by the China Scholarship Council (CSC) and in part under Project Agreement no. DSOCL10004 with DSO National Laboratories, Singapore.

\section{References}

[1] M. J. Sidi, Spacecraft Dynamics and Control: A Practical Engineering Approach, Cambridge Aerospace Series, Cambridge university press, New York, NY, USA, 1997.

[2] B. Wie and C. T. Plescia, "Attitude stabilization of flexible spacecraft during station-keeping maneuvers," Journal of Guidance, Control, and Dynamics, vol. 7, no. 4, pp. 430-436, 1984.

[3] T. C. Anthony, B. Wie, and S. Carroll, "Pulse-modulated control synthesis for a flexible spacecraft," Journal of Guidance, Control, and Dynamics, vol. 13, no. 6, pp. 1014-1022, 1990.

[4] B. N. Agrawal, "Spacecraft vibration suppression using smart structure," in Proceedings of the 4th International Congress on Sound and Vibration, pp. 563-570, St. Petersburg, Russia, 1996.

[5] H. Khalil, Nonlinear System, Prentice Hall, Upper Saddle River, NJ, USA, 2002. 
[6] R. L. Bagley and R. A. Calico, "Fractional order state equations for the control of viscoelastically damped structures," Journal of Guidance, Control, and Dynamics, vol. 14, no. 2, pp. 304-311, 1991.

[7] C. M. Ionescu, J. A. T. Machado, and R. De Keyser, "Modeling of the lung impedance using a fractional-order ladder network with constant phase elements," IEEE Transactions on Biomedical Circuits and Systems, vol. 5, no. 1, pp. 83-89, 2011.

[8] I. Podlubny, "Fractional-order systems and $P I^{\lambda} D^{\mu}$-controllers," Institute of Electrical and Electronics Engineers. Transactions on Automatic Control, vol. 44, no. 1, pp. 208-214, 1999.

[9] A. Oustaloup, J. Sabatier, and P. Lanusse, "From fractal robustness to the CRONE control," Fractional Calculus \& Applied Analysis, vol. 2, no. 1, pp. 1-30, 1999.

[10] C. A. Monje, Y. Chen, B. M. Vinagre, D. Xue, and V. Feliu, Fractional-Order Systems and Controls, Springer, New York, NY, USA, 2010.

[11] Y. Luo and Y. Chen, "Fractional order [proportional derivative] controller for a class of fractional order systems," Automatica, vol. 45, no. 10, pp. 2446-2450, 2009.

[12] M. S. Tavazoei and M. Haeri, "Describing function based methods for predicting chaos in a class of fractional order differential equations," Nonlinear Dynamics, vol. 57, no. 3, pp. 363-373, 2009.

[13] F. B. Duarte and J. T. MacHado, "Fractional describing function of systems with Coulomb friction," Nonlinear Dynamics, vol. 56, no. 4, pp. 381-387, 2009.

[14] B. Wie, Space Vehicle Dynamics and Control, AIAA Educational Series, Washington, DC, USA, 1998.

[15] Q. Hu and G. Ma, "Variable structure control and active vibration suppression of flexible spacecraft during attitude maneuver," Aerospace Science and Technology, vol. 9, no. 4, pp. 307-317, 2005.

[16] G. Song, N. V. Buck, and B. N. Agrawal, "Spacecraft vibration reduction using pulse-width pulse-frequency modulated input shaper," Journal of Guidance, Control, and Dynamics, vol. 22, no. 3, pp. 433-440, 1999.

[17] K. H. Kienitz and J. Bals, "Pulse modulation for attitude control with thrusters subject to switching restrictions," Aerospace Science and Technology, vol. 9, no. 7, pp. 635-640, 2005.

[18] T. D. Krovel, Optimal tuning of PWPF modulator for attitude control [M.S. thesis], Norwegian University of Science and Technology, 2005.

[19] Y. Q. Chen, I. Petráš, and D. Xue, "Fractional order controla tutorial," in Proceedings of the American Control Conference (ACC '09), pp. 1397-1411, St. Louis, Mo, USA, June 2009.

[20] I. Petráś, "Fractional-order memristor-based Chua's circuit," IEEE Transactions on Circuits and Systems II: Express Briefs, vol. 57, no. 12, pp. 975-979, 2010. 


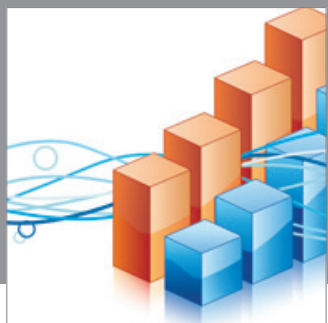

Advances in

Operations Research

mansans

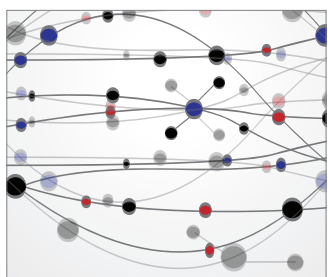

The Scientific World Journal
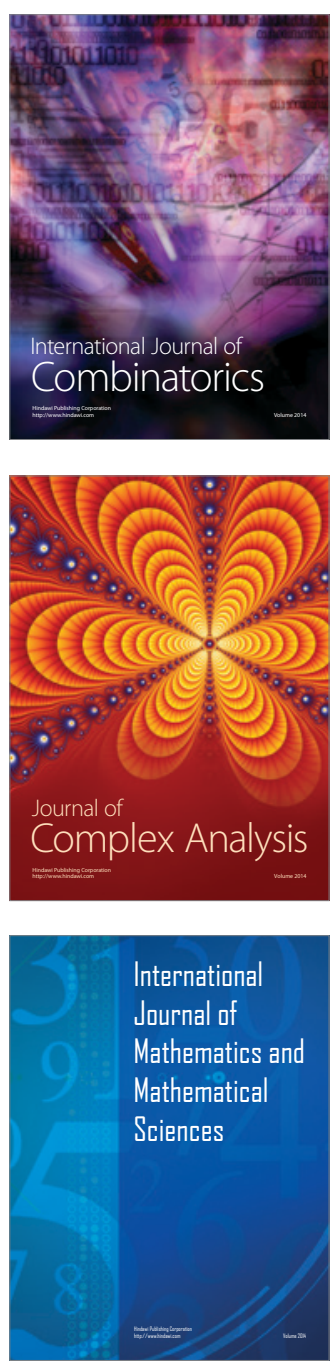
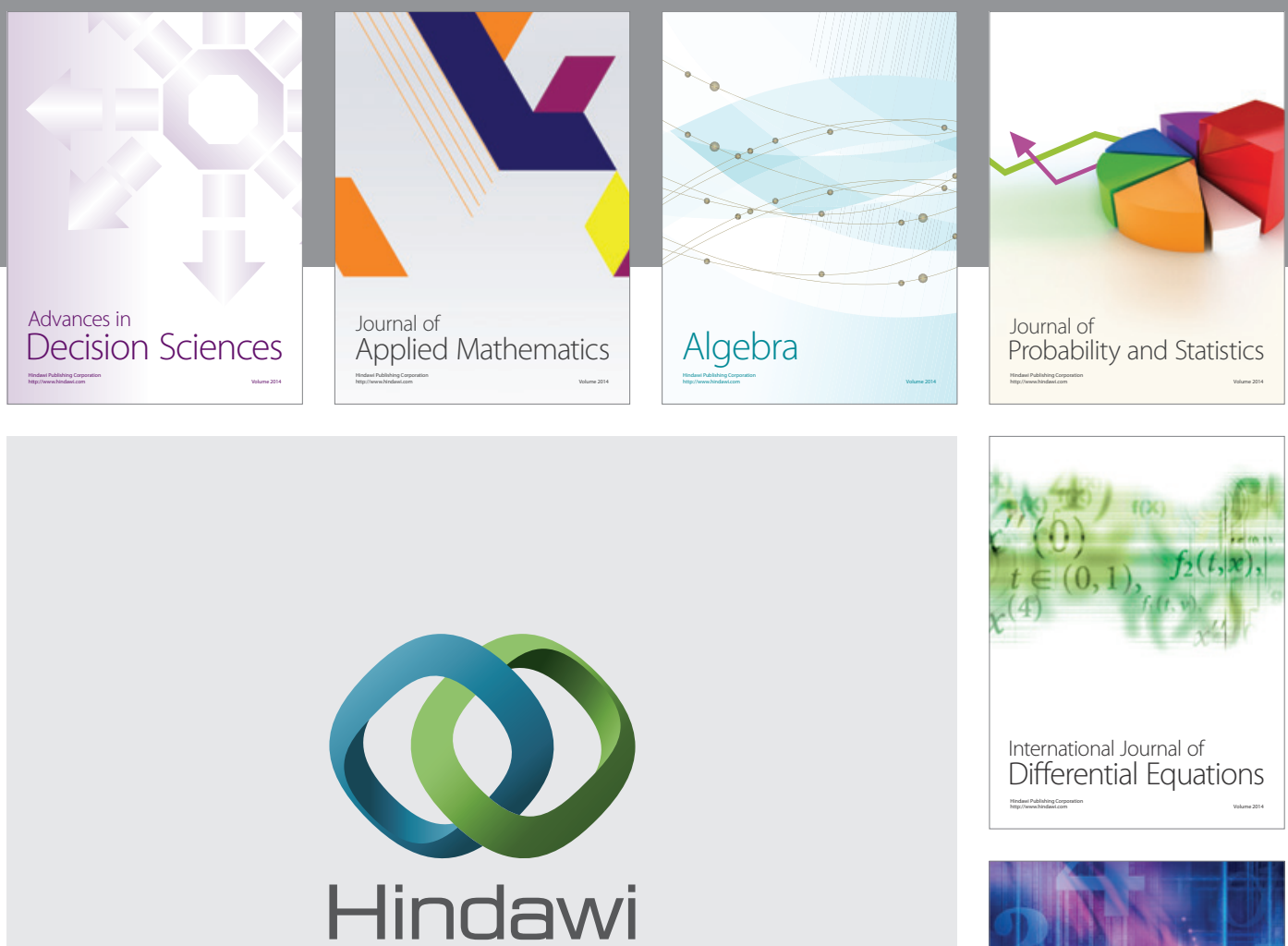

Submit your manuscripts at http://www.hindawi.com
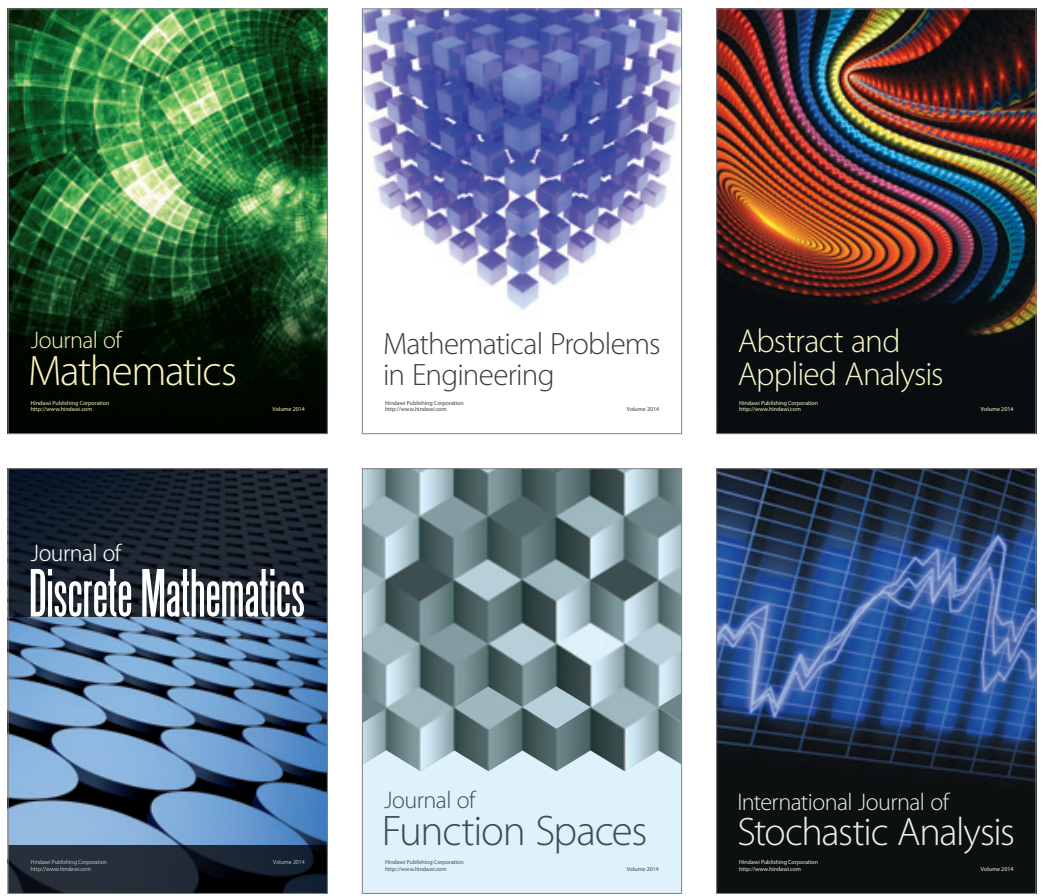

Journal of

Function Spaces

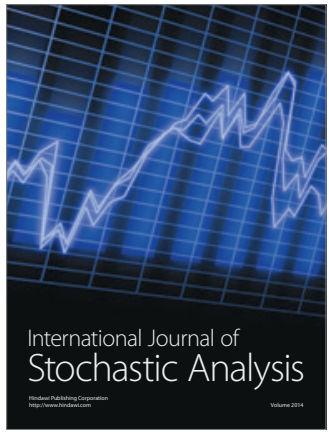

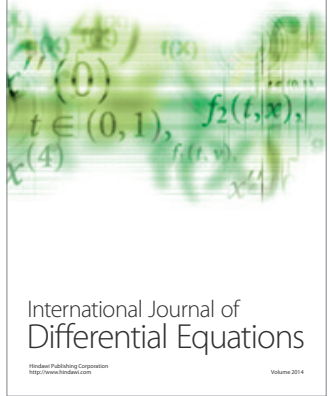
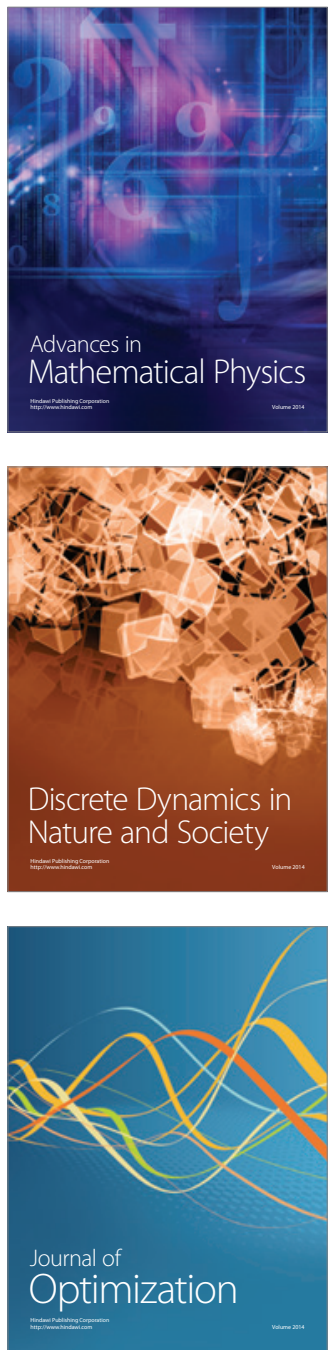